\title{
Chronotropic incompetence can limit exercise tolerance in COPD patients with lung hyperinflation
}

\author{
This article was published in the following Dove Press journal: \\ International Journal of COPD \\ 17 October 2016 \\ Number of times this article has been viewed
}

\section{Sébastien Hulo' \\ Jocelyn Inamo² \\ Aurélie Dehon ${ }^{3}$ \\ Olivier Le Rouzic ${ }^{4}$ \\ Jean-Louis Edme' \\ Remi Neviere ${ }^{5}$}

'Université Lille, CHU Lille, EA 4483, IMPact de l'Environnement Chimique sur la Santé humaine, Lille, France; ${ }^{2}$ Département de Cardiologie - CHU Fort de France, Martinique - Faculté de Médecine - Université des Antilles, France; ${ }^{3}$ Pôle d'Anesthésie Réanimation ADRU, CHU Nîmes, Nîmes, France; ${ }^{4}$ Université Lille, CNRS, Inserm, CHU Lille, Institut Pasteur de Lille, Center for Infection and Immunity of Lille, Lille, France; ${ }^{5}$ Université Lille, Inserm, CHU Lille, Lille Inflammation Research International Center, Lille, France
Correspondence: Sébastien Hulo Université de Lille - IMPECS - IMPact de l'Environnement Chimique sur la Santé Humaine (EA 4483), Faculté de Médecine de Lille - Pôle Recherche - $2^{\text {ème Étage }}$ Aile EST I, Place Verdun 59045 Lille Cedex, France

$\mathrm{Tel}+33320627761$

Fax +33 320627784

Email sebastien.hulo@univ-lille2.fr
Purpose: Metabolic-chronotropic relationship is the only concept that assesses the entire chronotropic function during exercise, as it takes into account individual fitness. To better understand interrelationships between chronotropic incompetence (CI), dynamic hyperinflation (DH) and exercise limitation among Global initiative for chronic Obstructive Lung Disease (GOLD) stages of chronic obstructive pulmonary disease (COPD) disease severity, we evaluated cardiopulmonary responses to symptom-limited cycle exercise in stable patients.

Patients and methods: We prospectively studied 47 COPD patients classified by GOLD stage severity. Pulmonary function tests and cardiopulmonary responses to symptom-limited incremental exercise were studied. CI was defined by regression line between percent heart rate $(\mathrm{HR})$ reserve and percent oxygen uptake $\left(V^{\prime} \mathrm{O}_{2}\right)$ reserve, ie, chronotropic-metabolic index (CMI). DH was defined from the knot resulting from the nonlinear regressions of inspiratory capacity changes from rest to peak (dynamic inspiratory capacity $\left(\mathrm{IC}_{\mathrm{dyn}}\right)$ ) with percentage of maximal HR and CMI.

Results: Aerobic capacity (median interquartile ranges) peak $V^{\prime} \mathrm{O}_{2}, 24.3$ (23.6; 25.2), 18.5 $(15.5 ; 21.8), 17.5(15.4 ; 19.1) \mathrm{mL} \cdot \mathrm{kg}^{-1} \cdot \mathrm{min}^{-1}$ and CMI worsened according to GOLD severity. The optimal knot of $\mathrm{IC}_{\mathrm{dyn}}$ was equal to $-0.34 \mathrm{~L}$. The multivariate logistic regression showed a strong relationship between CI (outcome) and DH (odds ratio [confidence interval 95]) 25 (3.5; 191.6).

Conclusion: COPD patients with DH have a poor cardiovascular response to exercise, which may be attributed to CI.

Keywords: COPD, hyperinflation, chronotropic incompetence, exercise

\section{Introduction}

Patients with chronic obstructive pulmonary disease (COPD) have limited exercise tolerance, which has been related to reduced maximal voluntary ventilation (MMV), impaired diffusion capacity of the lung and lung hyperinflation. ${ }^{1-3}$ The major consequence of lung hyperinflation is the association of increased ventilatory workload and decreased inspiratory muscle pressure generating capacity, which contributes to dyspnea and poor exercise tolerance. ${ }^{2,4}$ Beyond the lungs, deleterious cardiovascular consequences of dynamic hyperinflation (DH) have been consistently identified in COPD patients during incremental exercise. ${ }^{5-7}$ In these observations, DH was associated with reduced oxygen $\left(\mathrm{O}_{2}\right)$ uptake efficiency slope and $\mathrm{O}_{2}$ pulse, which noninvasively reflect cardiac stroke volume during submaximal exercise. ${ }^{6,8,9}$ Interestingly, lung volume reduction surgery has been shown to improve exercise tolerance through cardiac stroke volume increases. ${ }^{10}$

Along with cardiac stroke volume impairment is the concept that a contributing factor of reduced cardiac output leading to poor exercise tolerance might be the 
limitation to increase heart rate (HR), so called chronotropic incompetence (CI). ${ }^{11}$ Chronotropic regulation involves cardiac interactions with the autonomic nervous system, which influences HR response to exercise, HR recovery and multiple components of HR variability. ${ }^{12,13}$ HR increases upon initiation of exercise are principally mediated by the withdrawal of cardiac parasympathetic activity, with the sympathetic contribution being manifested at a longer latency. Since vagal withdrawal with the initiation of exercise can result to an increase of 30 to 50 beats per minute (BPM) in HR, further increases are thought to be related to sympathetic activation. Recovery involves reactivation of the parasympathetic system and deactivation of sympathetic activity, causing a decline in HR. Interestingly, both impaired HR increases in proportion to the metabolic demand and HR recovery kinetics are associated with poor outcome. ${ }^{13}$

An inability of HR to increase appropriately in proportion to the metabolic demands of exercise has been termed $\mathrm{CI}$, which has been originally defined as an inability to reach $80 \%$ of age-predicted maximum $\mathrm{HR}\left(\mathrm{HR}_{\max }\right)$ or HR reserve utilization. ${ }^{11}$ More adequately, the only concept that allows assessment of the entire chronotropic function is metabolicchronotropic relationship, which takes into account individual fitness. Indeed, to ensure that reduced peak HR is not solely related to low exercise capacity, CI must be defined by the regression line between percent $\mathrm{HR}$ reserve and percent oxygen uptake $\left(V^{\prime} \mathrm{O}_{2}\right)$ reserve, ie, chronotropic-metabolic index (CMI). ${ }^{11,14}$ Using this approach, reduced CMI has clearly identified subgroups of patients largely dependent upon an increased HR for an increase in cardiac output during incremental exercise (HR becoming the limiting factor). ${ }^{11}$ Furthermore, in heart failure patients, CI has been consistently associated with increased mortality, independently of confounding factors, such as age, gender, physical fitness, and traditional cardiovascular risk factors. ${ }^{15,16}$

Mechanisms of CI are thought to be secondary to chronic overactivation of the sympathetic system and subsequent downregulation of cardiac $\beta$-adrenoreceptor densities. ${ }^{17,18}$ Consistent studies have shown that acute lung hyperinflation in breath-hold maneuvers can induce pulmonary vessels and heart compression leading to sympathetic activity increase. ${ }^{19,20}$ It is thus likely that chronic hyperinflation in COPD patients would lead to chronic sympathetic overactivation and blunted cardiac chronotropic response..$^{21,22}$ Recent findings supported that $\mathrm{CI}$ is common in patients with severe COPD and may be improved after lung volume reduction surgery. ${ }^{23,24} \mathrm{CI}$ was also found as an independent and powerful outcome predictor in severe COPD patients. ${ }^{25}$ Of note, whether CI as evaluated by CMI was related to lung hyperinflation has not been reported previously. Our aim was thus to test whether CI could be related to lung DH in exercising patients. We also aimed to determine the different factors, such as breathing pattern, $\mathrm{DH}$ and $\mathrm{CI}$, which can influence exercise intolerance in COPD patients.

\section{Patients and methods}

\section{Patients}

This study was conducted in accordance with the amended Declaration of Helsinki (http://www.wma.net/ en/30publications/10policies/b3/). The Institutional Review Board of Lille University Hospital (Lille, France) approved this study (2014-0111-b), which only included patients referred to our department for routine functional evaluation. Written informed consent was obtained from all patients. We prospectively enrolled all consecutive patients suffering from COPD, defined according to Global Initiative for Chronic Obstructive Lung Disease (GOLD) criteria. ${ }^{26}$ The diagnosis of COPD was confirmed by forced spirometry, when the patient was considered clinically stable. All the procedures and their risks were explained to the patients, who gave their verbal informed consent to enter the study. Pulmonary function testing as well as exercise studies had been undertaken for clinical reasons at the request of the patients' clinicians.

\section{Inclusion and exclusion criteria}

Caucasian subjects, either current or former smokers, with spirometrically confirmed COPD in GOLD stages were included. They had to have been free of COPD exacerbations during the last 3 months prior to inclusion. Patients with a history of left ventricular disease, left ventricular ejection fraction $<45 \%$, severe pulmonary hypertension (mean pulmonary artery pressure (PAP) $>35 \mathrm{mmHg}$ ) were excluded. Patients treated for hypertension with blood pressure $>160 / 90 \mathrm{mmHg}$, with arrhythmias, with significant coronary artery disease or using $\beta$-blockers were also excluded. None of the subjects had noninvasive positive-pressure ventilation support or long-term ambulatory $\mathrm{O}^{2}$ treatment.

\section{Pulmonary function}

Standard forced expiratory spirometry (forced expiratory volume in the first second $\left(\mathrm{FEV}_{1}\right)$ and forced expiratory vital capacity (FVC)) and body plethysmography (residual volume (RV), functional residual capacity (FRC), total lung capacity (TLC)), and diffusing capacity of the lung for carbon monoxide $\left(D_{\mathrm{L}, \mathrm{CO}}\right)$ were performed (MasterScreen ${ }^{\mathrm{TM}}$ body plethysmograph, Viasys, France) using European and American Thoracic Society guidelines. ${ }^{27}$ 


\section{Cardiopulmonary testing}

Cardiopulmonary exercise testing was performed according to standardized procedures using an electromagnetic braked cycle ergometer. ${ }^{28}$ Exercise protocol involved an initial 2 minutes of rest, followed by 2 minutes of unloaded cycling with a progressive increment every minutes $\left(10 \mathrm{~W} \cdot \mathrm{m}^{-1}\right)$ until exhaustion at a pedaling frequency of $60-65 \mathrm{rpm}$. Subjects were continuously monitored by 12-lead electrocardiogram (Cardiosoft, CareFusion, France). Blood pressure assessed by sphygmomanometry was recorded every 2 minutes. Arterial blood $(240 \mu \mathrm{L})$ was sampled at rest and at peak exercise and immediately analyzed using a blood gas analyzer/cooximeter (ABL700, Radiometer, France).

Subjects respired through an oro-nasal mask (Hans Rudolf 7450 SeriesV2 ${ }^{\text {TM }}$ Mask, CareFusion, France). Breath-bybreath cardiopulmonary data (Vyntus, CareFusion, France) were measured at rest, warm up and incremental exercise testing. Before each test, $\mathrm{O}_{2}$ and carbon dioxide $\left(\mathrm{CO}_{2}\right)$ analyzers and flow mass sensor were calibrated using available precision gas mixture and a 3-L syringe, respectively. Minute ventilation $\left(V_{\mathrm{E}}^{\prime}\right), V^{\prime} \mathrm{O}_{2}$, carbon dioxide output $\left(V^{\prime} \mathrm{CO}_{2}\right)$ were recorded as concurrent 10 seconds moving averages, as was determined ventilation anaerobic threshold (AT) by the V-slope method. Ventilatory reserve was calculated as $\left(\mathrm{MVV}\right.$ - peak $\left.V_{\mathrm{E}}{ }_{\mathrm{E}}\right) / \mathrm{MVV} * 100$ where MVV is maximal voluntary ventilation estimated as $\mathrm{FEV}_{1}$ multiplied by 35 . Peak values were averaged over the last 30 seconds of exercise. Patient effort was considered to be maximal if two of the following occurred: predicted maximal work is achieved, predicted maximal $\mathrm{HR}$ is achieved, $V_{\mathrm{E}}^{\prime} / V^{\prime} \mathrm{O}_{2}>45$, lactate level $>6 \mathrm{mmol} \cdot \mathrm{L}^{-1}$, RER $>1.10$ and $\mathrm{pH}$ drop $>0.06$, as recommended by the ATS/ACCP. ${ }^{28}$ At peak exercise, subjects assessed Borg-perceived exertion ratings for both respiratory and leg discomfort. During the study period, mean values between qualified replicate tests performed weekly on control subjects were $5.1 \% \pm 4.2 \%, 5.4 \% \pm 3.2 \%, 6.1 \% \pm 2.2 \%$, for peak $V^{\prime} \mathrm{O}_{2}, V^{\prime} \mathrm{CO}_{2}$ and $V_{\mathrm{E}}^{\prime}$, respectively.

Peak oxygen pulse $\left(\mathrm{O}_{2}\right.$ pulse $)$ was calculated and was expressed in $\mathrm{mL}$ per beat and as percentage of predicted value by dividing the predicted peak $V^{\prime} \mathrm{O}_{2}$ by predicted peak HR. $V_{\mathrm{E}}^{\prime} / V^{\prime} \mathrm{CO}_{2}$ slope was calculated off-line as a linear regression function using 10 -s averaged values and excluding the non-linear part of the relationship after the respiratory compensation point (where nonlinear rise in $V_{\mathrm{E}}$, occurred relative to $V^{\prime} \mathrm{CO}_{2}$ in the presence of decrease of end-tidal pressure of $\mathrm{CO}_{2}$ ). The $V^{\prime} \mathrm{O}_{2}$ efficiency slope (OUES) describes the relationship between $V^{\prime} \mathrm{O}_{2}$ and $V_{\mathrm{E}}^{\prime}$ during incremental exercise, via a log transformation of $V_{\mathrm{E}} \cdot{ }^{29}$ OUES was expressed as the slope of the linear relationship of $\log _{10} V_{\mathrm{E}}$, to $V^{\prime} \mathrm{O}_{2}\left(\mathrm{~L} \cdot \mathrm{min}^{-1}\right)$ using $V^{\prime} \mathrm{O}_{2}\left(\mathrm{~L} \cdot \mathrm{min}^{-1}\right)=\mathrm{m}\left(\log _{10} V^{\prime} \mathrm{E}\right)+\mathrm{b}$, where $\mathrm{m}=$ OUES. Double-product ( $\mathrm{mmHg} \cdot \mathrm{bpm})$ was calculated as the product of systolic blood pressure by the HR.

\section{$\mathrm{DH}$}

Changes in operational lung volumes were evaluated from measurements of inspiratory capacity (IC). ${ }^{1-3}$ End-expiratory lung volume (EELV) was assessed from IC maneuvers at rest, every 2 minutes during exercise and at peak exercise (Vyntus). In these maneuvers, after EELV was observed to be stable over 3-4 breaths, subjects were instructed to inspire maximally to TLC. For each measurement, EELV was calculated as resting TLC minus IC, using the plethysmographic TLC value. Dynamic $\mathrm{IC}\left(\mathrm{IC}_{\mathrm{dyn}}\right)$ was defined as resting IC minus IC at peak exercise. $\mathrm{DH}$ as dichotomous variable was performed from optimal knot of $\mathrm{IC}_{\mathrm{dyn}}$ (see section "Statistical analysis", "Results", and Figure 1). An inflection

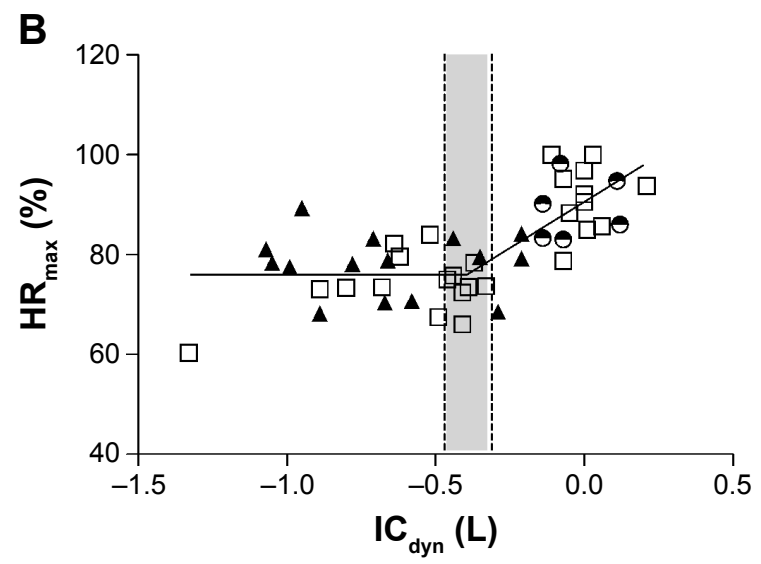

Figure I Relationship between dynamic inspiratory capacity $\left(\mathrm{IC}_{\mathrm{dyn}}\right)$ changes from rest to peak with chronotropic-metabolic index and percentage of age-predicted maximum heart rate $\left(\mathrm{HR}_{\max }\right),(\mathbf{A}$ and $\mathbf{B})$, respectively.

Notes: Global Initiative for Chronic Obstructive Lung Disease stages (stage I: half-full dots; stage 2: empty squares; stage 3 and 4: filled triangles). The broken-line trend is represented by the black solid lines. 
in tidal volume $\left(V_{\mathrm{T}}\right)$ relative to $V_{\mathrm{E}}^{\prime}$ (expressed as \% peak $V_{\mathrm{E}}^{\prime}$ ) was determined in each patient during exercise.

\section{$\mathrm{CMI}$}

Chronotropic index was defined as an index of maximal predicted HR reserve achieved. ${ }^{11}$ The percentage of HR reserve used at peak exercise referred to $\left[\left(\mathrm{HR}_{\text {stage }}-\mathrm{HR}_{\text {rest }}\right) /(220-\right.$ age in years $\left.\left.-\mathrm{HR}_{\text {rest }}\right)\right] * 100$, where $\mathrm{HR}$ is heart rate. For any given stage of exercise, the percentage of $V^{\prime} \mathrm{O}_{2}$ reserve used referred to $\left[\left(V^{\prime} \mathrm{O}_{2 \text { stage }}-V^{\prime} \mathrm{O}_{2 \text { rest }}\right) /\left(V^{\prime} \mathrm{O}_{2 \text { peak }}-V^{\prime} \mathrm{O}_{2 \text { rest }}\right)\right] *$ 100. CMI was calculated off-line as the regression line slope between percent $\mathrm{HR}$ reserve and percent $V^{\prime} \mathrm{O}_{2}$ reserve. A patient was referred to as having $\mathrm{CI}$ if the resulting slope index was $<0.75$ and $\mathrm{HR}_{\max }<80 \%$. ${ }^{11,14}$

\section{Statistical analysis}

The statistical analyses were performed with SAS software (SAS Institute Inc., Cary, NC, USA; version 9.3). A twosided significance level of 0.05 was chosen for all tests. Descriptive statistics, with tests of normality (ShapiroWilk test) for values showed free distributions. Values were expressed as medians with interquartile ranges (IQR). Patients were divided according to GOLD classification. ${ }^{30}$ Nonparametric analysis (Kruskal-Wallis test) was used to test for differences between classes. Post hoc comparisons were determined by Wilcoxon test with Bonferroni correction. Relationships between quantitative variables were assessed by the Spearman's correlation coefficient (r). Bivariate comparisons for categorical variables were performed with Chi-squared test (with Fisher correction as appropriate).

Locally estimated scatterplot smoother SAS procedure showed a broken-line trend between CMI or $\mathrm{HR}_{\max }$ and $\mathrm{IC}_{\mathrm{dyn}}$ changes. Linear mixed model procedure was used to analyze the change of CMI and $\mathrm{HR}_{\text {max }}$ versus a broken function of $\mathrm{IC}_{\mathrm{dyn}}$ that took 0 before the knot and subject's $\mathrm{IC}_{\mathrm{dyn}}$ after the knot; the knot position was fixed by the smallest Akaike information criterion (AIC) using Gauss-Newton method. ${ }^{31,32}$ Thus, mean and $95 \%$ confidence interval of optimal knot, intercept (no change), and slope (increase) were estimated by nonlinear mixed model. As these two variables are used interchangeably in the literature to define CI, we took the middle of the overlap area of the $\mathrm{IC}_{\mathrm{dyn}}$ knot from these two relationships. $\mathrm{IC}_{\mathrm{dyn}}$ converted to $\mathrm{DH}$ as dichotomous variables indicating $\mathrm{IC}_{\mathrm{dyn}}<$ optimal knot $(\mathrm{DH}=1)$ or $\mathrm{IC}_{\mathrm{dyn}} \geq \operatorname{optimal} \operatorname{knot}(\mathrm{DH}=0)$. The association between $\mathrm{CI}$ and $\mathrm{DH}$ with covariables (age, body mass index (BMI), TLC, IC/TLC, RV/TLC, GOLD stage) was estimated as an odds ratio (OR) and $95 \%$ confidence intervals by using multivariate logistic regression analyses.

\section{Results}

We included 47 consecutive stable COPD patients (age 55 $(49 ; 61)$ years, BMI $26(22 ; 30)$, female $n=14)$. All patients were maximally treated with bronchodilators. At study entry, patients were receiving inhaled steroids (75\%), long-acting $\beta_{2}$-agonists (75\%) and tiotropium (65\%); all of them were active or ex-smokers. Overall, a wide range of airflow obstruction $\left(\mathrm{FEV}_{1} / \mathrm{FVC}\right.$ from $26 \%$ to $69 \% ; 56(50 ; 64) \%$ ), static hyperinflation (IC/TLC from $24 \%$ to $61 \%$ predicted; $40(36 ; 46) \%$ predicted $)$, diffusing capacity $\left(D_{\mathrm{L}, \mathrm{CO}}\right.$ from $17 \%$ to $98 \%$ predicted; $73(49 ; 85) \%$ predicted) was found. Nine out of 42 patients (21\%) suffered from well-controlled arterial hypertension and were on diuretics (72\%), ACE-inhibitors (52\%), calcium-antagonists (36\%) to control the disease.

According to objective ATS/ACCP criteria, exercise was considered maximal in all patients. ${ }^{28}$ Mean peak workload and $V^{\prime} \mathrm{O}_{2}$ was $90(65 ; 95)$ watts (range $55-170$ watts) and $18.5(15.6 ; 22.6) \mathrm{mL} \cdot \mathrm{kg}^{-1} \cdot \mathrm{min}^{-1}(67(61 ; 78) \%$ predicted $)$, respectively. AT $V^{\prime} \mathrm{O}_{2}$ was $12.1(11.0 ; 14.4) \mathrm{mL} \cdot \mathrm{kg}^{-1} \cdot \mathrm{min}^{-1}$. Peak $V_{\mathrm{T}}$ to $\mathrm{FEV}_{1}$ ratio was $37(30 ; 48) \%$ and plateau of $V_{\mathrm{T}}$ increase occurred at $70.8(61.1 ; 77.8) \%$ of maximum $V_{\mathrm{E}}$. Breathing reserve at peak exercise was reduced $(24(3 ; 33) \%)$. $\mathrm{IC}_{\mathrm{dyn}}$ change measured from rest to peak exercise was -0.39 $(-0.67 ;-0.07)$ L and peak EELV (\% TLC) was $64(56 ; 72) \%$. $\mathrm{HR}_{\max }$ was $134(119 ; 150) \mathrm{bpm}(80(73 ; 88) \%$ predicted $)$ with HR reserve utilization of $60(43 ; 76) \%$. Peak $\mathrm{O}_{2}$ pulse was $10.2(8.6 ; 12.4) \mathrm{mL} \cdot \mathrm{bpm}^{-1}(84(71 ; 97) \%$ predicted $)$. Peak systemic arterial partial pressure of $\mathrm{O}_{2}\left(\mathrm{~Pa}, \mathrm{O}_{2}\right)$ and $\mathrm{CO}_{2}\left(\mathrm{~Pa}, \mathrm{CO}_{2}\right)$ were $91(70 ; 99)$ and $39(35 ; 41) \mathrm{mmHg}$, respectively. Arterial lactate was $5.6(4.7 ; 7.0) \mathrm{mmol} \cdot \mathrm{L}^{-1}$ at peak exercise.

Functional characteristics of patients classified by GOLD scaling system are shown in Table 1. Static hyperinflation significantly decreased incrementally from stage I to stage III-IV among baseline pulmonary function parameters (Table 1). Tables 2-4 (main features of CPET, ventilatory and cardiovascular response details, respectively) display all results and significant differences of medians of exercise parameters between each group (GOLD stage I, stage II and stage III-IV) after adjustments. Peak $V^{\prime} \mathrm{O}_{2}$ (\% of predicted values) was not significantly different between GOLD stages (Table 2). At peak exercise (Table 3), EELV (\% TLC), $V_{\mathrm{D}} / V_{\mathrm{T}}(\%)$ and $\mathrm{Pa}, \mathrm{CO}_{2}$ significantly increased incrementally with patient's COPD severity. $\mathrm{IC}_{\mathrm{dyn}}$ significantly decreased incrementally with patient's COPD severity (Table 3 ).

The nonlinear procedure established between $\mathrm{IC}_{\mathrm{dyn}}$ change and CMI (Figure 1A) or $\mathrm{HR}_{\max }$ (Figure 1B) has converged to broken-line models. The best fit, which was defined 
Table I Subject characteristics

\begin{tabular}{|c|c|c|c|c|}
\hline GOLD stage & Stage I & Stage II & Stage III-IV & $P$-value \\
\hline Sample size & $n=6$ & $n=26$ & $n=15$ & \\
\hline Age, years & $53.5(49 ; 56)$ & $60(54 ; 64)^{\ddagger}$ & $49(44 ; 56)^{\ddagger}$ & 0.031 \\
\hline $\mathrm{BMI}, \mathrm{kg} \cdot \mathrm{m}^{-2}$ & $26.0(23.8 ; 26.4)$ & $27.8(22.8 ; 30.7)$ & $21.9(18.9 ; 29.1)$ & 0.224 \\
\hline Gender, $M / F$ & $4 / 2$ & $22 / 4$ & $7 / 8$ & 0.028 \\
\hline Smoking status, \% current & 50 & 40 & 20 & 0.238 \\
\hline Smoking, pack-year & $30(30 ; 40)$ & $27.5(16.5 ; 40)$ & $42.5(25 ; 6 I)$ & 0.279 \\
\hline \multicolumn{5}{|l|}{ Lung function test } \\
\hline $\mathrm{FEV}_{1}, \mathrm{~L}$ & $3.2(3.0 ; 3.4)^{*, \#}$ & $2.1(1.8 ; 2.6)^{*, \neq}$ & $1.4(1.2 ; 1.7)^{\#, \neq}$ & $<0.001$ \\
\hline $\mathrm{FEV}_{1}$, \% predicted & $0.88(0.83 ; 0.94)^{*, \#}$ & $0.66(0.59 ; 0.72)^{*, \neq}$ & $0.45(0.34 ; 0.48)^{\#, \neq}$ & $<0.001$ \\
\hline FVC, L & $4.9(4.4 ; 5.7)^{* \#}$ & $3.8(3.1 ; 4.3)^{*, \neq}$ & $3.1(2.7 ; 3.6)^{\# . \neq}$ & $<0.001$ \\
\hline $\mathrm{FEV}_{\mathrm{I}} / \mathrm{FVC}, \%$ & $67.2(64.8 ; 68.4)^{*, \#}$ & $58.8(54.1 ; 63.7)^{*, \neq}$ & $47.8(32.6 ; 54.5)^{\#, \neq}$ & $<0.001$ \\
\hline IC, L & $3.2(3.0 ; 4.4)^{\#}$ & $2.8(2.4 ; 3.3)^{\ddagger}$ & $2.1(1.9 ; 2.7)^{\# . \neq}$ & 0.001 \\
\hline TLC, L & $7.4(6.7 ; 8.1)$ & $6.1(5.6 ; 7.3)$ & $6.5(5.8 ; 7.5)$ & 0.187 \\
\hline FRC, L & 3.7 (3.6; 3.9) & $3.5(3.0 ; 4.6)$ & $4.5(3.5 ; 4.8)$ & 0.248 \\
\hline IC/TLC & $45.7(40.8 ; 53.8)^{\#}$ & $4 I .5(38.8 ; 47.1)^{\ddagger}$ & $35.6(29.4 ; 39.9)^{\#, \neq}$ & $<0.001$ \\
\hline $\mathrm{RV}, \mathrm{L}$ & $2.4(2.3 ; 2.9)^{\#}$ & $2.8(1.8 ; 3.4)$ & $3.4(2.7 ; 4.0)^{\#}$ & 0.012 \\
\hline RV/TLC, \% & $98(86 ; 100)^{*, \#}$ & II $4.5(104 ; 123)^{*, \neq}$ & $145(136 ; 160)^{\#, \neq}$ & $<0.001$ \\
\hline$D_{\mathrm{L}, \mathrm{CO}}, \mathrm{mL} \cdot \mathrm{min}^{-1} \cdot \mathrm{mmHg}^{-1}$ & $23.0(15.2 ; 26.7)$ & $19.8(14.4 ; 24.3)$ & $11.6(9.6 ; 15.5)$ & 0.032 \\
\hline$D_{\mathrm{L}, \mathrm{CO}}, \%$ predicted & $81.5(59 ; 88)$ & $75(5 \mathrm{I} ; 85)$ & $48(38 ; 62)$ & 0.033 \\
\hline
\end{tabular}

Notes: Data are presented as median (interquartile range). *\#: Significant difference between groups. Bold values represent the significant results.

Abbreviations: GOLD, Global Initiative for Chronic Obstructive Lung Disease; BMI, body mass index; FEV , forced expiratory volume in the first second; FVC, forced expiratory vital capacity; IC, inspiratory capacity; TLC, total lung capacity; FRC, functional residual capacity; RV, residual volume; $D_{L, c o}$, diffusing capacity of the lung for carbon monoxide; M, male; F, female.

by the smallest AIC, allowed us to obtain the values of the following knots $\mathrm{IC}_{\mathrm{dyn}}$ (mean \pm standard error): $-0.28 \pm 0.1 \mathrm{~L}$ and $-0.39 \pm 0.08 \mathrm{~L}$, respectively. The middle of the overlap area allowed us to determine the $\mathrm{DH}$ threshold with $\mathrm{IC}_{\mathrm{dyn}}$ change equal to $-0.34 \mathrm{~L}$.

The prevalence of DH was higher in GOLD III-IV patients than in GOLD I-II patients (Table 5). Patients with lung hyperinflation had more CI than patients without DH (Table 5). CI status was not related to GOLD score $(P=0.1)$.

The logistic regression that analyzed the association between CI (outcome) and DH showed a strong relationship (OR [confidence interval 95\%]) 20 [4.4-91.9] that was confirmed by multivariate analysis taking into account the following covariates: age, BMI, TLC, CI/TLC, RV/TLC and GOLD stage (Table 6).

\section{Discussion}

A new finding of this study is that lung hyperinflation in COPD patients is associated with impaired metabolic-chronotropic relationship, so-called CI, which may present as a limiting factor of exercise tolerance.

In our study, both static and dynamic lung volume measurements were performed to assess lung hyperinflation in COPD patients. Among baseline pulmonary function parameters, we used IC/TLC ratio as a static hyperinflation-derived variable

Table 2 Cardiopulmonary exercise testing (peak values, except AT)

\begin{tabular}{|c|c|c|c|c|}
\hline GOLD stage & Stage I & Stage II & Stage III-IV & $P$-value \\
\hline Sample size & $n=6$ & $n=26$ & $n=\mid 5$ & \\
\hline Workload, W & $97.5(95 ; 150)^{\#}$ & $90(80 ; 100)^{\ddagger}$ & $65(60 ; 85)^{\#, \neq}$ & $<\mathbf{0 . 0 0 1}$ \\
\hline $\mathrm{V}^{\prime} \mathrm{O}_{2}, \mathrm{~mL} \cdot \mathrm{kg}^{-1} \cdot \mathrm{min}^{-1}$ & $24.3(23.6 ; 25.2)^{\#}$ & $18.5(15.5 ; 21.8)$ & $17.5(15.4 ; 19.1)^{\#}$ & 0.035 \\
\hline$V^{\prime} \mathrm{O}_{2}, \%$ predicted & $77.4(62.8 ; 80.9)$ & 70.0 (55.0; 80.0) & $64.0(61.0 ; 68.0)$ & 0.215 \\
\hline AT $\mathrm{V}^{\prime} \mathrm{O}_{2}, \mathrm{~mL} \cdot \mathrm{kg}^{-1} \cdot \mathrm{min}^{-1}$ & $16.0(15.0 ; 16.6)^{* \#}$ & I2.I $(10.6 ; 14.1)^{*}$ & I2.0 (I I.0; I3.4) & 0.033 \\
\hline RER & I.2 (I.0; I.3) & I.2 (I.I; I.2) & I.2 (I.I; I.2) & 0.859 \\
\hline Arterial lactate, $\mathrm{mmol} \cdot \mathrm{L}^{-1}$ & $7.6(5.7 ; 9.2)$ & $5.7(4.8 ; 7.3)$ & $5.5(4.3 ; 6.4)$ & 0.036 \\
\hline Borg dyspnea score & $4(3 ; 7)$ & $4(3 ; 5)$ & $3(3 ; 5)$ & 0.865 \\
\hline Borg leg effort score & $4(4 ; 7)$ & $4(3 ; 5)$ & $3(2 ; 5)$ & 0.314 \\
\hline
\end{tabular}

Notes: Data are presented as median (interquartile range). ${ }^{*}, \#$, Significant difference between groups. Bold values represent the significant results.

Abbreviations: AT, anaerobic threshold; GOLD, Global Initiative for Chronic Obstructive Lung Disease; V $\mathrm{O}_{2}$, oxygen uptake; RER, respiratory exchange ratio. 
Table 3 Ventilatory response during submaximal exercise (peak values, except $V_{\mathrm{T}} / V_{\mathrm{E}}$ inflexion point)

\begin{tabular}{|c|c|c|c|c|}
\hline GOLD stage & Stage I & Stage II & Stage III-IV & $P$-value \\
\hline Sample size & $n=6$ & $n=26$ & $n=15$ & \\
\hline$f_{R}$, breaths $\cdot \min ^{-1}$ & $3 I(23 ; 4 I)$ & $31(27 ; 38)$ & $34(30 ; 38)$ & 0.655 \\
\hline$V_{T}, \mathrm{~L}$ & $2.3(1.8 ; 3.3)^{\#}$ & $1.8(1.6 ; 2.0)^{\ddagger}$ & $1.5(I . I ; I .6)^{\#, \neq}$ & 0.001 \\
\hline$V_{T} / \mathrm{FEV}_{1}, \%$ & $74.9(59.5 ; 91.0)^{\#}$ & $83.6(74.6 ; 91.0)^{\ddagger}$ & $105.0(85.6 ; 1 \mid 7.2)^{\#, \neq}$ & 0.003 \\
\hline$V_{T} / F V C, \%$ & $49.5(40.7 ; 57.3)$ & $48.4(45.2 ; 5 I .1)$ & $46.3(38.5 ; 50.2)$ & 0.649 \\
\hline$V_{E}^{\prime}, L \cdot m^{-1}$ & $76.5(64 ; 88)^{*, \#}$ & $54.5(48 ; 64)^{*, \ddagger}$ & $48(37 ; 53)^{\#, \ddagger}$ & $<0.001$ \\
\hline$V_{\mathrm{E}}^{\prime}$ reserve, \% predicted & $33.9(11.7 ; 44.9)^{\#}$ & $28.0(21.8 ; 34.4)^{\ddagger}$ & $2.7(-10.5 ; 16.6)^{\#, . \neq}$ & $<0.001$ \\
\hline$V_{\mathrm{T}} / V_{\mathrm{E}}^{\prime}$ inflection point, \% peak $V_{\mathrm{E}}$ & $52.5(46 ; 61)^{* . \#}$ & $40(32 ; 50)^{*, \neq}$ & $30(26 ; 32)^{\#, \neq}$ & $<0.001$ \\
\hline$I C_{\text {dyn }}, \mathrm{L}$ & $-0.08(-0.14 ; 0.11)^{\#}$ & $-0.38(-0.52 ; 0)^{\ddagger}$ & $-0.67(-0.95 ;-0.35)^{\# . \neq}$ & $<0.001$ \\
\hline EELV/TLC, \% & $53(49 ; 58)^{\#}$ & $61(54 ; 70)^{\ddagger}$ & $70(68 ; 79)^{\#, \neq}$ & $<0.001$ \\
\hline \multicolumn{5}{|l|}{ Gas exchange } \\
\hline$V_{\mathrm{D}} / V_{\mathrm{T}}, \%$ & $32(3 \mathrm{I} ; 35)^{\#}$ & $37(31 ; 40)^{\ddagger}$ & $45(38 ; 5 I)^{\#, \neq}$ & 0.004 \\
\hline $\mathrm{Pa}, \mathrm{O}_{2}, \mathrm{mmHg}$ & $99.8(98 ; 101)$ & $93.5(80.7 ; 99)$ & $86(60 ; 93)$ & 0.065 \\
\hline $\mathrm{Pa}, \mathrm{CO}_{2}, \mathrm{mmHg}$ & $33.1(27.0 ; 35.0)^{* . \#}$ & $38.5(36.0 ; 40.6)^{*, \ddagger}$ & $4 I .4(40.7 ; 45.0)^{\#, \neq}$ & $<0.001$ \\
\hline
\end{tabular}

Notes: Data are presented as median (interquartile range). *.\#.:Significant difference between groups. Bold values represent the significant results.

Abbreviations: GOLD, Global Initiative for Chronic Obstructive Lung Disease; $f_{\mathrm{R}}$, breathing frequency; $V_{\mathrm{T}}$, tidal volume; FEV ${ }_{1}$, forced expiratory volume in the first second; $F V C$, forced expiratory vital capacity; $V_{E}$, minute ventilation; $I_{\text {dyn }}$, dynamic inspiratory capacity; EELV, end-expiratory lung volume; TLC, total lung capacity; $V_{D}$, dead volume; $\mathrm{Pa}, \mathrm{O}_{2}$, arterial partial pressure of oxygen; $\mathrm{Pa}, \mathrm{CO}_{2}$, arterial partial pressure of carbon dioxide.

reflecting mechanical inspiratory constraint. ${ }^{1-3}$ We found that over a wide range of airflow, IC/TLC decreased incrementally with $\mathrm{FEV}_{1} / \mathrm{FVC}$ obstruction. Such relationship was also observed with RV/TLC, which increased incrementally with $\mathrm{FEV}_{1} / \mathrm{FVC}$. Next, we established exercise-induced DH by measuring $\mathrm{IC}_{\text {dyn }} \cdot{ }^{1-3} \mathrm{IC}_{\text {dyn }}$ significantly decreased incrementally with patient's GOLD score severity. DH was associated with increased $V_{\mathrm{T}}$ constraint as $V_{\mathrm{T}} / V^{\prime}$, inflection point expressed as peak $V_{\mathrm{E}}$, percent was decreased incrementally with patient's COPD severity. DH was accompanied with poor cardiovascular response, which was evaluated in our study by noninvasive surrogate markers, such as OUES and $\mathrm{O}_{2}$ pulse..$^{5-7} \mathrm{O}_{2}$ pulse, a noninvasive measure of stroke volume, requires assumption that the arteriovenous $\mathrm{O}_{2}$ content difference remains constant during exercise. Physiologically, arterial $\mathrm{O}_{2}$ content can be altered by hemoglobin availability, arterial blood oxygenation and peripheral $\mathrm{O}_{2}$ extraction. ${ }^{33}$ In our series of COPD patients, hemoglobin concentration and calculated arterial content were within the normal ranges. Furthermore, whether $\mathrm{O}_{2}$ extraction was normal or reduced in our patients, these changes may not account for $\mathrm{O}_{2}$ pulse reduction, since a lower $\mathrm{O}_{2}$ extraction would actually increase $\mathrm{O}_{2}$ pulse. ${ }^{6,10}$

Beside the negative effects of abnormal ventilatory mechanics on cardiac stroke volume, $\mathrm{CI}$ can also prevent the

Table 4 Cardiovascular response during submaximal exercise (peak values, except recovery)

\begin{tabular}{|c|c|c|c|c|}
\hline GOLD stage & Stage I & Stage II & Stage III-IV & $P$-value \\
\hline Sample size & $n=6$ & $\mathrm{n}=\mathbf{2 6}$ & $n=15$ & \\
\hline $\mathrm{HR}$, beat $\cdot \mathrm{min}^{-1}$ & $149(142 ; 162)$ & $130(1|3 ;| 50)$ & $133(122 ; 140)$ & 0.074 \\
\hline HR peak-rest, beat $\cdot \mathrm{min}^{-1}$ & $60(54 ; 7 I)$ & $42.5(32 ; 67)$ & $42(28 ; 61)$ & 0.102 \\
\hline $\mathrm{HR}_{\max }, \%$ & $88.2(83.3 ; 94.7)^{\#}$ & $79.2(73.4 ; 90.6)$ & $78.8(70.7 ; 83.2)^{\#}$ & 0.042 \\
\hline HR reserve use, \% & $76.3(67.5 ; 87.3)^{\#}$ & $55.0(41.3 ; 80.6)$ & $52.5(42.6 ; 63.8)^{\#}$ & 0.032 \\
\hline Chronotropic-metabolic index & $0.68(0.6 \mathrm{I} ; 0.73)^{\#}$ & $0.58(0.47 ; 0.75)$ & $0.47(0.39 ; 0.60)^{\#}$ & 0.027 \\
\hline $\mathrm{O}_{2}$ pulse, $\mathrm{mL} \cdot$ beat $^{-1}$ & II.7 $(10.5 ; 12.4)^{\#}$ & $10.8(10.1 ; 13.9)^{\ddagger}$ & $8.3(7.3 ; 9.7)^{\# .+}$ & $<0.001$ \\
\hline $\mathrm{O}_{2}$ pulse, $\%$ predicted & $95.5(88.0 ; 104.0)^{\#}$ & $86.0(74.0 ; 99.0)^{\ddagger}$ & $77.0(65.0 ; 82.0)^{\#, \ddagger}$ & 0.005 \\
\hline OUES, $\mathrm{mL} \cdot \mathrm{min}^{-1}$ & I 548 (I 344; I 988) & I 70 I (| 467; I 988) & | 32I (I |38; I 937) & 0.188 \\
\hline$V_{E}^{\prime} / V^{\prime} \mathrm{CO}_{2}$ slope & $26.1(23.8 ; 30.3)$ & $30.2(26.9 ; 33.5)$ & $32.0(29.0 ; 37.4)$ & 0.079 \\
\hline Mean arterial pressure, $\mathrm{mmHg}$ & $125.3(120.0 ; 133.0)$ & $123.7(103.5 ; 135.2)$ & $117.0(111.7 ; 125.0)$ & 0.537 \\
\hline Double product, $\mathrm{mmHg} \cdot$ beat $\cdot \mathrm{min}^{-1}$ & $29523(28 \text { I } 20 ; 3 \mid 266)^{\#}$ & $25619(23$ 47I; 29 539)‡ & $20295(19380 ; 24$ 024) \#, & $<0.001$ \\
\hline \multicolumn{5}{|l|}{ Post-exercise heart rate recovery } \\
\hline HR recovery at I $\mathrm{min}$, beat $\cdot \mathrm{min}^{-1}$ & $17.5(13 ; 18)$ & $10(3 ; 19)$ & $13(6 ; 20)$ & 0.636 \\
\hline $\mathrm{HR}$ recovery at $3 \mathrm{~min}$, beat $\cdot \mathrm{min}^{-1}$ & $4 I(31 ; 49)$ & $28.5(20 ; 35)$ & $27(19 ; 36)$ & 0.262 \\
\hline
\end{tabular}

Notes: Data are presented as median (interquartile range). \#, Significant difference between groups. Bold values represent the significant results.

Abbreviations: GOLD, Global Initiative for Chronic Obstructive Lung Disease; HR, heart rate; $\mathrm{HR}_{\max }$, age predicated maximum heart rate; min, minute; $\mathrm{O}_{2}$, oxygen; OUES, oxygen uptake efficiency slope; $V_{E}^{\prime}$, minute ventilation; $V^{\prime} \mathrm{CO}_{2}$, carbon dioxide output. 
Table 5 Relationships between dynamic hyperinflation, GOLD stage and chronotropic incompetence

\begin{tabular}{llll}
\hline $\begin{array}{l}\text { Group } \\
\text { patients }\end{array}$ & $\begin{array}{l}\text { No } \\
\text { hyperinflation* }\end{array}$ & Hyperinflation* & P-value** \\
\hline GOLD & & 14 & $\mathbf{0 . 0 1 9}$ \\
I-II & 18 & 12 & \\
III-IV & 3 & 76.9 & $<\mathbf{0 . 0 0 0 I}$ \\
Chronotropic & 14.3 & & \\
incompetence, \% & & & \\
\hline
\end{tabular}

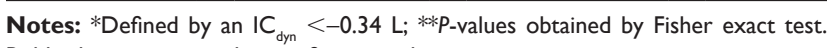
Bold values represent the significant results.

Abbreviations: GOLD, Global Initiative for Chronic Obstructive Lung Disease; $I_{\mathrm{dyn}}$, dynamic inspiratory capacity.

cardiovascular system from reaching its physiological limits before maximal exercise. ${ }^{11}$ In healthy humans, HR increases virtually instantaneously upon the initiation of exercise. Involvement of a reduction in cardiac parasympathetic activity has been demonstrated in human studies showing that atropine can significantly attenuate the initial increase in HR in a variety of exercise conditions. In contrast, the magnitude of HR increase at the onset of exercise is not diminished by prior $\beta$-adrenergic blockade, implying a minimal influence of sympathetic activity at this time..$^{34,35}$ Thereafter, there is an approximately linear relationship between HR and $V^{\prime} \mathrm{O}_{2}$ during incremental dynamic exercise. At moderateto-elevated exercise intensities $\beta$-adrenergic blockade significantly attenuates the size of the HR response, thus suggesting a key role for heightened sympathetic nervous system and elevated catecholamine levels in the circulation. ${ }^{34-36}$ At immediate cessation of exercise, HR abruptly declines, which is typically followed by a more gradual decline of HR over minutes depending on exercise conditions. Initial HR recovery is related to rapid restoration of cardiac parasympathetic activity, since it is virtually abolished by atropine administration, but unaffected by $\beta$-adrenergic blockade. ${ }^{37}$

Blunted HR response is the primary cause of or a significant contributor to severe, symptomatic exercise intolerance

Table 6 Multivariate analysis of chronotropic incompetence

\begin{tabular}{ll}
\hline Variables & OR (95\% confidence interval) \\
\hline Dynamic hyperinflation status & $\mathbf{2 5}(\mathbf{3 . 5}-191.6) *$ \\
Age & $1.094(1.008-1.187) *$ \\
BMI & $0.952(0.826-1.098)$ \\
TLC & $0.676(0.342-1.336)$ \\
IC/TLC & $0.956(0.849-1.076)$ \\
RV/TLC & $1.004(0.970-1.039)$ \\
GOLD stage & $1.056(0.088-12.634)$ \\
\hline
\end{tabular}

Notes: $* P$-values $<0.05$. Bold values represent the significant results. Abbreviations: OR, odds ratio; BMI, body mass index; TLC, total lung capacity; IC, inspiratory capacity; RV, residual volume; GOLD, Global Initiative for Chronic Obstructive Lung Disease. in heart failure patients. ${ }^{15,16}$ Mechanisms of CI have been related to overstimulation of the sympathetic system, which induces downregulation of $\beta$-receptors and their desensitization in the presence of increased circulating catecholamine levels. ${ }^{17,18}$ In COPD patients, a striking elevation of sympathetic tone has been demonstrated and might thus provide a link between COPD and CI. Hypoxemia, chemoreflexes, impaired baroreflex sensitivity, muscle ergoreflex and lung hyperinflation are important factors contributing to neurohumoral activation in COPD. ${ }^{20,38}$ In normal subjects, sympathetic nerve firings are synchronized with central inspiratory motor activity and increase in lung volume during inspiration activates pulmonary vagal afferents that in turn inhibit sympathetic nerve discharge. ${ }^{20,38}$ The relative magnitude of these two opposing mechanisms determines the net effect of respiratory modulation of sympathetic nerve activity, which is in favor of sympathetic activation in COPD patients..$^{21,22}$

Although lung hyperinflation may induce sympathetic overactivation in COPD patients, whether DH would lead to CI during submaximal exercise has not been previously tested. In normal subjects, $\mathrm{CI}$ has been most commonly diagnosed when HR fails to reach $80 \%-85 \%$ of the $\mathrm{HR}_{\max }{ }^{11}$ However, before one concludes that a patient has $\mathrm{CI}$, it is important to consider the patient's level of effort. ${ }^{14}$ Whether attenuated HR in response to incremental exercise is related to low effort level or CI represents a major issue in this context. Hence, in order to more objectively evaluate $\mathrm{CI}$, the relationship between $\mathrm{HR}$ and $V^{\prime} \mathrm{O}_{2}$ during exercise should be measured. ${ }^{11,14}$ In this approach, the metabolic-chronotropic relationship is calculated from the ratio of the HR reserve to the metabolic reserve during submaximal exercise. ${ }^{39,40}$ If the assumption on which percentage of $\mathrm{HR}$ reserve equal $V^{\prime} \mathrm{O}_{2}$ reserve is achieved in normal adults, a relationship slope less than $0.75-0.8$ is considered indicative of $\mathrm{CI}$ in chronic heart disease patients. ${ }^{39,40} \mathrm{In}$ our study, this metabolic-chronotropic relationship approach allowed us to define an association between $\mathrm{CI}$ and $\mathrm{DH}$, which was further characterized by broken-line trend analysis with a reduced $\mathrm{IC}_{\mathrm{dyn}}$ of $0.34 \mathrm{~L}$ as a threshold level for CI.

In conclusion, we have shown that CI evaluated by the metabolic-chronotropic relationship is highly prevalent in a cohort of COPD patients. Adding evaluation of CI to standard pulmonary function parameters at rest and during incremental exercise allows to determine the level of lung hyperinflation as a potential mechanism of attenuated HR response.

\section{Acknowledgment}

The authors thank all of the staff of the Pulmonary Functional Tests Department for their help in this work. 


\section{Author contributions}

All authors contributed toward data analysis, drafting and revising the paper and agree to be accountable for all aspects of the work.

\section{Abbreviations}

ACE inhibitor, angiotensin-converting-enzyme inhibitor; AIC, Akaike information criterion; AT, anaerobic threshold; ATS/ACCP, American Thoracic Society/American College of Chest Physicians; BMI, body mass index; CI, chronotropic incompetence; CMI, chronotropic-metabolic index; COPD, chronic obstructive pulmonary disease; CPET, cardiopulmonary exercise testing; $\mathrm{DH}$, dynamic hyperinflation; $D_{\mathrm{L}, \mathrm{CO}}$, diffusing capacity of the lung for carbon monoxide; EELV, end-expiratory lung volume; $\mathrm{FEV}_{1}$, forced expiratory volume in the first second; FRC, functional residual capacity; FVC, forced expiratory vital capacity; GOLD, Global Initiative for Chronic Obstructive Lung Disease; $\mathrm{HR}$, heart rate; $\mathrm{HR}_{\max }$, age predicted maximum heart rate; $\mathrm{IC}$, inspiratory capacity; $\mathrm{IC}_{\text {dyn }}$, dynamic inspiratory capacity; IQR, interquartile ranges; MVV, maximal voluntary ventilation; $\mathrm{O}_{2}$ pulse, peak oxygen pulse; OUES, oxygen uptake efficiency slope; $\mathrm{Pa}$, arterial partial pressure; PAP, pulmonary artery pressure; RER, respiratory exchange ratio; RV, residual volume; TLC, total lung capacity; $V^{\prime} \mathrm{CO}_{2}$, carbon dioxide output; $V_{\mathrm{E}}$, minute ventilation; $V^{\prime} \mathrm{O}_{2}$, oxygen uptake; $V_{\mathrm{D}}$, death volume; $V_{\mathrm{T}}$, tidal volume.

\section{Disclosure}

The authors report no conflicts of interest in this work.

\section{References}

1. Gagnon P, Guenette JA, Langer D, et al. Pathogenesis of hyperinflation in chronic obstructive pulmonary disease. Int JChron Obstruct Pulmon Dis. 2014;9:187-201

2. Guenette JA, Webb KA, O’Donnell DE. Does dynamic hyperinflation contribute to dyspnoea during exercise in patients with COPD? Eur Respir J. 2012;40(2):322-329.

3. Langer D, Ciavaglia CE, Neder JA, Webb KA, O’Donnell DE. Lung hyperinflation in chronic obstructive pulmonary disease: mechanisms, clinical implications and treatment. Expert Rev Respir Med. 2014;8(6): 731-749.

4. Vogiatzis I, Zakynthinos S. Factors limiting exercise tolerance in chronic lung diseases. Compr Physiol. 2012;2(3):1779-1817.

5. Chuang ML, Huang SF, Su CH. Cardiovascular and respiratory dysfunction in chronic obstructive pulmonary disease complicated by impaired peripheral oxygenation. Int J Chron Obstruct Pulmon Dis. 2015;10: 329-337.

6. Tzani P, Aiello M, Elia D, et al. Dynamic hyperinflation is associated with a poor cardiovascular response to exercise in COPD patients. Respir Res. 2011;12:150.

7. Visca D, Aiello M, Chetta A. Cardiovascular function in pulmonary emphysema. Biomed Res Int. 2013;2013:184678.
8. Teopompi E, Tzani P, Aiello M, et al. Fat-free mass depletion is associated with poor exercise capacity irrespective of dynamic hyperinflation in COPD patients. Respir Care. 2014;59(5):718-725.

9. Vassaux C, Torre-Bouscoulet L, Zeineldine S, et al. Effects of hyperinflation on the oxygen pulse as a marker of cardiac performance in COPD. Eur Respir J. 2008;32(5):1275-1282.

10. Lammi MR, Ciccolella D, Marchetti N, Kohler M, Criner GJ. Increased oxygen pulse after lung volume reduction surgery is associated with reduced dynamic hyperinflation. Eur Respir J. 2012;40(4): 837-843.

11. Brubaker PH, Kitzman DW. Chronotropic incompetence: causes, consequences, and management. Circulation. 2011;123(9):1010-1020.

12. Fisher JP, Young CN, Fadel PJ. Autonomic adjustments to exercise in humans. Compr Physiol. 2015;5(2):475-512.

13. Coote JH. Recovery of heart rate following intense dynamic exercise. Exp Physiol. 2010;95(3):431-440.

14. Wilkoff BL, Miller RE. Exercise testing for chronotropic assessment. Cardiol Clin. 1992;10(4):705-717.

15. Benes J, Kotrc M, Borlaug BA, et al. Resting heart rate and heart rate reserve in advanced heart failure have distinct pathophysiologic correlates and prognostic impact: a prospective pilot study. JACC Heart Fail. 2013;1(3):259-266.

16. Wang J, Fang F, Yip GW, et al. Importance of chronotropic response and left ventricular long-axis function for exercise performance in patients with heart failure and preserved ejection fraction. Int J Cardiol. 2016;202:339-343.

17. Bristow MR, Hershberger RE, Port JD, et al. Beta-adrenergic pathways in nonfailing and failing human ventricular myocardium. Circulation. 1990;82(2 Suppl):I12-I25.

18. Colucci WS, Ribeiro JP, Rocco MB, et al. Impaired chronotropic response to exercise in patients with congestive heart failure. Role of postsynaptic beta-adrenergic desensitization. Circulation. 1989; $80(2): 314-323$.

19. Breskovic T, Steinback CD, Salmanpour A, Shoemaker JK, Dujic Z. Recruitment pattern of sympathetic neurons during breath-holding at different lung volumes in apnea divers and controls. Auton Neurosci. 2011; 164(1-2):74-81.

20. Dempsey JA, Sheel AW, St Croix CM, Morgan BJ. Respiratory influences on sympathetic vasomotor outflow in humans. Respir Physiol Neurobiol. 2002;130(1):3-20.

21. Andreas S, Anker SD, Scanlon PD, Somers VK. Neurohumoral activation as a link to systemic manifestations of chronic lung disease. Chest. 2005;128(5):3618-3624.

22. van Gestel AJ, Kohler M, Clarenbach CF. Sympathetic overactivity and cardiovascular disease in patients with chronic obstructive pulmonary disease (COPD). Discov Med. 2012;14(79):359-368.

23. Armstrong HF, Gonzalez-Costello J, Jorde UP, et al. The effect of lung volume reduction surgery on chronotropic incompetence. Respir Med. 2012;106(10):1389-1395.

24. Gupta M, Bansal V, Chhabra SK. Abnormal heart rate recovery and chronotropic incompetence on exercise in chronic obstructive pulmonary disease. Chron Respir Dis. 2013;10(3):117-126.

25. González-Costello J, Armstrong HF, Jorde UP, et al. Chronotropic incompetence predicts mortality in severe obstructive pulmonary disease. Respir Physiol Neurobiol. 2013;188(2):113-118.

26. Gupta N, Pinto LM, Morogan A, Bourbeau J. The COPD assessment test: a systematic review. Eur Respir J. 2014;44(4):873-884.

27. Miller MR, Hankinson J, Brusasco V, et al. Standardisation of spirometry. Eur Respir J. 2005;26(2):319-338.

28. American Thoracic Society; American College of Chest Physicians. ATS/ACCP Statement on cardiopulmonary exercise testing. Am J Respir Crit Care Med. 2003;167(2):211-277.

29. Hollenberg M, Tager IB. Oxygen uptake efficiency slope: an index of exercise performance and cardiopulmonary reserve requiring only submaximal exercise. J Am Coll Cardiol. 2000;36(1):194-201. 
30. Rabe KF, Hurd S, Anzueto A, et al. Global strategy for the diagnosis, management, and prevention of chronic obstructive pulmonary disease: GOLD executive summary. Am J Respir Crit Care Med. 2007;176(6): $532-555$

31. Robbins KR, Saxton AM, Southern LL. Estimation of nutrient requirements using broken-line regression analysis. J Anim Sci. 2006;84 Suppl: E155-E165.

32. Nève V, Edmé JL, Matran R. Earlier decline in sniff nasal inspiratory pressure than peak expiratory flow in children with Duchenne muscular dystrophy. Eur Respir J. 2014;44(5):1361-1363.

33. Whipp BJ, Higgenbotham MB, Cobb FC. Estimating exercise stroke volume from asymptotic oxygen pulse in humans. J Appl Physiol (1985). 1996;81(6):2674-2679.

34. Robinson BF, Epstein SE, Beiser GD, Braunwald E. Control of heart rate by the autonomic nervous system. Studies in man on the interrelation between baroreceptor mechanisms and exercise. Circ Res. 1966;19(2):400-411.

35. Fagraeus L, Linnarsson D. Autonomic origin of heart rate fluctuations at the onset of muscular exercise. J Appl Physiol. 1976;40(5):679-682.
36. Maciel BC, Gallo L Jr, Marin Neto JA, Lima Filho EC, Martins LE. Autonomic nervous control of the heart rate during dynamic exercise in normal man. Clin Sci (Lond). 1986;71(4):457-460.

37. Imai $\mathrm{K}$, Sato $\mathrm{H}$, Hori $\mathrm{M}$, et al. Vagally mediated heart rate recovery after exercise is accelerated in athletes but blunted in patients with chronic heart failure. J Am Coll Cardiol. 1994;24(6):1529-1535.

38. Jouett NP, Watenpaugh DE, Dunlap ME, Smith ML. Interactive effects of hypoxia, hypercapnia and lung volume on sympathetic nerve activity in humans. Exp Physiol. 2015;100(9):1018-1029.

39. da Cunha FA, Farinatti Pde T, Midgley AW. Methodological and practical application issues in exercise prescription using the heart rate reserve and oxygen uptake reserve methods. J Sci Med Sport. 2011;14(1) 46-57.

40. Mezzani A, Corrà U, Giordano A, Cafagna M, Adriano EP, Giannuzzi P. Unreliability of the $\% \mathrm{VO} 2$ reserve versus \%heart rate reserve relationship for aerobic effort relative intensity assessment in chronic heart failure patients on or off beta-blocking therapy. Eur J Cardiovasc Prev Rehabil. 2007;14(1):92-98.
International Journal of COPD

\section{Publish your work in this journal}

The International Journal of COPD is an international, peer-reviewed journal of therapeutics and pharmacology focusing on concise rapid reporting of clinical studies and reviews in COPD. Special focus is given to the pathophysiological processes underlying the disease, intervention programs, patient focused education, and self management protocols.

\section{Dovepress}

This journal is indexed on PubMed Central, MedLine and CAS. The manuscript management system is completely online and includes a very quick and fair peer-review system, which is all easy to use. Visit http://www.dovepress.com/testimonials.php to read real quotes from published authors.

Submit your manuscript here: http://www.dovepress.com/international-journal-of-chronic-obstructive-pulmonary-disease-journal 